\title{
Impact of crustal deformation detection by the DSI (difference of split-band interferograms) method with PALSAR-2 data: A case study on the 2016 Kumamoto Earthquake
}

\section{Taku Ozawa ( $\nabla$ taku@bosai.go.jp ) 2040-6572 \\ Yuji Himematsu \\ National Research Institute for Earth Science and Disaster Resilience}

National Research Institute for Earth Science and Disaster Resilience https://orcid.org/0000-0002-

\section{Express Letter}

Keywords: difference of split-band interferograms, InSAR, crustal deformation, the 2016 Kumamoto Earthquake

Posted Date: October 11th, 2021

DOI: https://doi.org/10.21203/rs.3.rs-952510/v1

License: (c) (1) This work is licensed under a Creative Commons Attribution 4.0 International License.

Read Full License 
1 Title: Impact of crustal deformation detection by the DSI (difference of split-band

2 interferograms) method with PALSAR-2 data: A case study on the 2016

3 Kumamoto Earthquake

4 Author \#1: Taku Ozawa, National Research Institute for Earth Science and Disaster

5 Resilience, 1-9 Tennodai, Tsukuba, Ibaraki, Japan, taku@bosai.go.jp

6 Author \#2: Yuji Himematsu, National Research Institute for Earth Science and Disaster

$7 \quad$ Resilience, 1-9 Tennodai, Tsukuba, Ibaraki, Japan, himematsu@ bosai.go.jp

8

9 The corresponding author: Taku Ozawa (taku@bosai.go.jp)

10

11 


\section{Abstract}

13 Interferometric Synthetic Aperture Radar (InSAR) is a useful tool for detecting

14 surface deformations at high spatial resolutions. When InSAR is applied to huge surface

15 deformations, clear fringes with complicated phase gaps often appear in the

16 interferograms. Although the surface deformations in such areas are important for

17 understanding their mechanisms and for investigating disasters, it is difficult to convert

18 the data on such fringes to surface deformation information because of difficulties

19 associated with phase unwrapping. To resolve these difficulties, we created multiple

20 SAR pairs with different frequencies using a band-pass filter and derived the difference

21 of interferograms which are generated from these SAR pairs. Generally, its result

22 corresponds to the result of SAR observations made with long-wavelength radar.

23 Therefore, a phase wrap was less likely to occur, and phase unwrapping was easy to

24 accomplish. We applied this method to the PALSAR-2 data pairs for the 2016

25 Kumamoto Earthquake and succeeded in identifying huge crustal deformations with

26 complicated phase gaps in the vicinity of surface ruptures. Comparing these results with

27 the crustal deformations observed from GNSS measurements, the root-mean-squares of 
28 the differences were found to be approximately $4 \mathrm{~cm}$. Although this accuracy was

29 poorer than that of conventional InSAR, it was nearly equivalent to that of the offset-

30 tracking method. Furthermore, its spatial resolution was significantly better than that of

31 the offset-tracking method. However, the disadvantage of this method is that its

32 detection accuracy is significantly degraded in zones with low coherence, due to noise

33 amplification. The standard deviation of the noise component was approximately $2 \mathrm{~cm}$

34 for pixels with coherences above 0.7 . However, for pixels with a coherence lower than

350.2 , the standard deviation was greater than $10 \mathrm{~cm}$, and the noise component

36 occasionally exceeded $1 \mathrm{~m}$. Despite the disadvantages of this method, it is effective for

37 the detection of huge crustal deformations with high spatial resolution in areas where

38 phase unwrapping methods for conventional InSAR are inappropriate.

39

$40 \quad$ Keywords

41 DSI, difference of split-band interferograms, InSAR, crustal deformation, the 2016

42 Kumamoto Earthquake 


\section{Introduction}

45 Interferometric Synthetic Aperture Radar (InSAR) is a useful tool for detecting the

46 surface deformations associated with earthquakes, volcanic activity, landsides, and so

47 on. In particular, the L-band SAR which is installed on the ALOS-2 (SAR sensor name:

48 PALSAR-2) and upcoming ALOS-4 and NISAR has been utilized as an effective tool

49 for detecting surface deformations in heavily vegetated areas. This is due to its capacity

50 to penetrate thick vegetation and its high stability of coherence. Another advantage of

51 the L-band SAR in InSAR applications is its robust capacity to detect huge

52 deformations with large spatial gradient. Generally, such huge deformation produces a

53 dense fringe, which makes it difficult to solve modulus $2 \pi$ ambiguities. Considering

54 simple 1-D phase unwrapping as an example, it is impossible to solve its ambiguities in

55 case where the phase gaps of neighboring pixels exceed a half cycle. Because the

56 wavelength of the L-band is longer than that of the C-and X-bands, such a phase gap is

57 unlikely to occur. Thus, it is relatively easy to detect huge deformations using L-band

58 SAR. However, when large earthquake events cause surface ruptures, even if a clear

59 fringe with high coherence is obtained, many phase gaps exceeding half a wavelength 
60 often appear in interferograms. In such cases, the phase information around the surface

61 rupture is not converted to the corresponding amount of crustal deformation. Figure 1

62 illustrates the interferogram depicting crustal deformation around the surface rupture

63 associated with the 2016 Kumamoto Earthquake (April 16, 2016, MJMA 7.3) derived

64 from PALSAR-2 data. Clear fringes are also seen near the fault, but their pattern is

65 extremely complicated, and many phase gaps exceeding a half cycle are apparent. It is

66 difficult to correctly unwrap the phases in this region. However, information about the

67 crustal deformations in such areas is important for improving the understanding

68 earthquake occurrence mechanisms, investigating damaged areas, and so on. Therefore,

69 it is meaningful to obtain surface deformation information for such areas. Himematsu

70 and Furuya (2016) applied the offset-tracking method to PALSAR-2 data and obtained

71 information on crustal deformation near the fault. Thus, the offset-tracking method is an

72 effective tool; however, a large correlation window is necessary for high accuracy, and

73 thus, there is a trade-off between detection accuracy and spatial resolution in this

74 method.

75 Many unwrapping algorithms for InSAR processing have been proposed. For 
76 instance, the Brunch-cut method, which is one of the basic algorithms, solves for

77 modulus $2 \pi$ ambiguities at each pixel while searching for an adequate path that connects

78 phases while considering residues and coherence (Goldstein et al., 1988). The phase

79 connection is processed such that the phase difference between adjacent pixels does not

80 exceed a half cycle. However, as mentioned before, huge crustal deformations often

81 cause phase gaps exceeding a half cycle, so it is difficult to obtain unwrapped phases in

82 areas surrounded by such phase gaps. Although many other advanced phase unwrapping

83 algorithms such as minimum cost flow and statistical cost flow have been proposed

84 (Constantini, 1998; Chen and Zebker, 2000), it remains difficult to unwrap phases in

85 such areas.

86 Madsen and Zebker (1992) proposed a method for deriving the unwrapped phase

87 from the phase differences of interferograms with different frequencies. Such

88 interferograms are derived from SAR pairs created by dividing the chirp band using the

89 band-pass filter, which is called "split-spectrum method." In the 1990s, there were

90 limited data sets with sufficiently high coherence for the application of the split-

91 spectrum method. Recently, L-band SAR with a wide bandwidth has become available, 
92 and a chance which is obtained interferograms with sufficiently high coherence has

93 drastically increased. Thus, the split-spectrum method has recently seen use as a general

94 technique to estimate ionospheric delay (Bricic et al., 2010; Rosen et al., 2010; Gomba

95 et al., 2016; Furuya et al., 2017; Wegmüller et al., 2018). Therefore, recent L-band

96 SAR data can also be useful for obtaining the unwrapped phase using the split-spectrum

97 method. While this is an application of the split spectrum method, in this paper, we refer

98 to it as the "DSI" (difference of split-band interferograms) to distinguish it from the

99 split-spectrum method used for the estimation of the ionospheric delay.

100 We applied the DSI method to PALSAR-2 data for the 2016 Kumamoto Earthquake

101 (Fig. 1), and succeeded in detecting huge crustal deformations near the fault. In this

102 paper, we describe the fundamentals of crustal deformation detection using the DSI

103 method with practical processing procedures, and discuss its detection accuracy,

104 advantages, and disadvantages.

105

106 The basics of the DSI method

107 In this section, we describe the fundamentals of the DSI method used to obtain the 


$$
\Delta \phi_{\mathrm{obs}}=4 \pi f / c \Delta \rho_{\text {non-disp }}-4 \pi K / c f \Delta \mathrm{TEC}+\text { bias }_{1}
$$

112 where $\Delta \rho_{\text {non-disp }}$ is the non-dispersive component of the slant-range change due to crustal

113 deformation, orbit difference, atmospheric delay, etc. The second term on the right-hand

114 side is called the dispersive component, which is mainly due to ionospheric delay. $f$ is

115 the radar frequency, $c$ is the speed of light, $\triangle \mathrm{TEC}$ is a total electron content along the

116 radar propagation path, $K$ is constant of $40.28 \mathrm{~m}^{3} / \mathrm{s}^{2}$, and bias 1 is the bias. First, it is

117 necessary to estimate and remove the dispersive component. From Eq. (1), the response

118 to frequency is different for dispersive and non-dispersive components. The split-

119 spectrum method produces interferograms with different frequencies from SAR images

120 that divide chirp bands, and divide the dispersive and nondispersive components based

121 on the differences in responses (Bricic et al., 2010; Rosen et al., 2010; Gomba et al.,

122 2016; Wegmüller et al., 2018). Next, the non-deformation components in the

123 nondispersive component are removed. The phase component of the orbit difference can

124 be estimated from the satellite ephemeris and a digital terrain model. In Japan, 
atmospheric delay can be estimated with an accuracy of several centimeters, from the

126 results of the numerical weather model published by the Japan Meteorological Agency

127 (JMA) (Ozawa and Shimizu, 2010; Ozawa et al., 2019). The deformation component

128 can be extracted by subtracting the non-deformation components from the initial

129 interferogram. However, it is a wrapped phase, and as described above, it is difficult to

130 obtain an appropriate unwrapped phase for huge crustal deformations with complicated

131 phase gaps. For example, in InSAR applications using the PALSAR-2 data (radar

132 wavelength: approximately $24 \mathrm{~cm}$ ), a slant-range change of approximately $12 \mathrm{~cm}$ causes

133 a one cycle phase change; a deformation gap exceeding $6 \mathrm{~cm}$ makes it difficult to obtain

134 the unwrapped phase. Such huge deformations often appear due to earthquakes,

135 volcanic activities, landslides, glaciers, and so on. Conversely, if a microwave with a

136 long wavelength is used, this problem is unlikely to occur. Then, we used the DSI

137 method to obtain the unwrapped phase. The DSI method analyzes interferograms of the

138 different frequencies generated in the ionospheric delay estimation. Subtracting

139 dispersive and non-deformation components from their interferograms, the phase

140 difference $\left(\Delta \phi_{\text {diff }}\right)$ is 


$$
\Delta \phi_{\text {diff }}=\Delta \phi_{\text {high }}-\Delta \phi_{\text {low }}=4 \pi \Delta \rho_{\text {deform }}\left(f_{\text {high }}-f_{\text {low }}\right) / c+\text { bias }_{2}
$$

144 where $f_{\text {low }}$ and $f_{\text {high }}$ are the center frequencies of SAR images divided into two bands:

$145 \Delta \phi_{\text {high }}$ and $\Delta \phi_{\text {low }}$ are the phase differences of the interferograms of high and low bands,

$146 \Delta \rho_{\text {deform }}$ is the slant-range change due to crustal deformation, and bias $_{2}$ is the bias. This

147 is consistent with the interferometric phase obtained from the SAR pair with a radar

148 frequency of $f_{\text {high }}-f_{\text {low. }}$. The wavelength of this interferogram was $c /\left(f_{\text {high }}-f_{\text {low }}\right)$. A phase

149 wrap does not occur when the slant-range change is less than a quarter of the

150 wavelength, and unwrapping is not necessary. The slant range change occasionally

151 exceeds its threshold, but even in such cases, the need to perform an unwrapping can be

152 eliminated by increasing the number of band divisions. This method is described in the

153 next section, along with an explanation of the actual procedure.

\section{Procedure of the DSI analysis: A case study on the 2016}

\section{Kumamoto Earthquake}

157 In this chapter, we describe the procedure for crustal deformation detection using the 
158 DSI method, with a case study on the 2016 Kumamoto Earthquake using PALSAR-2

159 data. The sequence of the 2016 Kumamoto Earthquake began from a foreshock

160 occurrence $\left(\mathrm{M}_{\mathrm{W} 6.1)}\right.$ at 21:26 on April 14, 2016 (JST) in the Kumamoto Prefecture of

161 the Kyushu district of western Japan. According to JMA, the depth of the hypocenter

162 was $11.4 \mathrm{~km}$. F-net, the broadband seismometer network of the National Research

163 Institute for Earth Science and Disaster Resilience (NIED) (Okada et al., 2004), derived

164 a lateral slip mechanism with the north-south tension axis at a depth of $17 \mathrm{~km}$. The

165 mainshock (Mw7.1) occurred two days later, on April 16 at 1:25 JST. F-net determined

166 the same lateral-slip mechanism as the foreshock, and the depth determined by the JMA

167 was $11 \mathrm{~km}$. In our previous study, we detected the crustal deformation associated with

168 the earthquake by applying conventional InSAR to PALSAR-2 data, and showed that its

169 result could be explained by the fault model, with right-lateral slips on three faults and

170 one normal fault with a low angle; three right-lateral faults were identified along the

171 Hinagu Fault, the Futagawa Fault, and its northeast extension, and the normal fault was

172 parallel to the Futagawa Fault (Ozawa et al., 2016). Dense fringes with complicated

173 phase gaps appeared (Fig. 1(d)) in the vicinity of the Futagawa Fault, where surface 
174 ruptures with dislocations exceeding $2 \mathrm{~m}$ were found (e.g., Shirahama et al., 2016, Toda

175 et al., 2016). Complicated phase gaps were also observed in the western section of the

176 Aso caldera, where significant stress changes were induced by the ruptures associated

177 with the mainshock. In our previous study, crustal deformation was not obtained in

178 these areas because of difficulties associated with phase unwrapping (Fig. 1(c)). Using

179 the detection of such complicated deformations as an example, we describe the

180 procedure of the DSI analysis.

181 In this analysis, we analyzed the PALSAR-2 data obtained from Path 28 (descending

182 orbit, left-looking) and Path 126 (ascending orbit, left-looking). Most of the scenes were

183 mountainous areas covered by vegetation. The observation mode was SM1 for both

184 paths. Its center frequency is $1.2575 \mathrm{GHz}$, and the bandwidth of the SLC image is 80

$185 \mathrm{MHz}$. The incidence angles were $32^{\circ}$ and $24^{\circ}$ at the scene center, respectively. The

186 observation dates were April 15, 2016, and April 29, 2016, for both paths, and

187 therefore, the results from their pairs did not include the crustal deformations associated

188 with the foreshock on April 14, 2016. In this analysis, we used RINC software, which is

189 an InSAR analysis tool developed by NIED (Ozawa et al., 2016). The number of looks 
190 in the interferogram generation were set to $11 \times 15$ for Path 28 and $8 \times 12$ for Path 126 so

191 that the ground range was from 25 to $30 \mathrm{~m}$. Although processing with a smaller number

192 of looks is possible, we adopt a larger number of looks for reduction of decorrelation

193 noise. The spectral shift filter described by Gatelli et al. (1994) was applied to the

194 interferogram generation, and the spectral enhancement filter described by Goldstein

195 and Werner (1998), with a filter coefficient of 0.8 and a window size of 32 pixels, was

196 applied to the generated interferograms. For the simulation of the phase component due

197 to topography, a numerical ellipsoidal height model created from a $10 \mathrm{~m}$ mesh digital

198 elevation model published by the Geospatial Information Authority of Japan (GSI) and

199 the EGM96 geoid model (Lemoine et al., 1997) was used.

200 Next, we estimated the ionospheric delay using the split-spectrum method. The

201 method by Brcic et al. (2010), Rosen et al. (2010), and Gomba et al. (2016) needs to

202 unwrap the phase including the crustal deformation component; therefore, it was

203 difficult to estimate the ionospheric delay near the fault, where unwrapping errors were

204 likely to occur. Thus, we used the method described by Wegmüller et al. (2018) to

205 extract the ionospheric delay component without unwrapping. This method creates two 
206 SLC image pairs with different frequencies by dividing the chirp band using a band-

207 pass filter. In the case dividing to two bands, the center frequencies of their SLC images

208 were $1.2375 \mathrm{GHz}\left(f_{\text {low }}\right)$ and $1.2775 \mathrm{GHz}\left(f_{\text {high }}\right)$, and the bandwidth was $40 \mathrm{MHz}$. The

209 dispersive component ( $\left.\Delta \phi_{\text {disp }}\right)$ was estimated as follows:

210

$2112 \Delta \phi_{\text {disp }}=\Delta \phi_{\text {obs }}-\Delta \phi_{\text {diff }} f_{\text {low }} f_{\text {high }} / f_{0} /\left(f_{\text {high }}-f_{\text {low }}\right)$

213 where $f_{0}$ is the center frequency of the full-band SLC image, and $\Delta \phi_{\text {diff }}$ is the phase

214 difference between interferograms for high and low bands, corresponding to the

215 interferometric phase observed by a radar wave with a frequency of $f_{\text {high }}-f_{\text {low. }}$ Its

216 frequency is $40 \mathrm{MHz}$, and the wavelength is approximately $7.5 \mathrm{~m}$ in this case study.

217 When this processing is applied to interferograms with the topographic and orbital

218 phase components removed, $\Delta \phi_{\text {diff }}$ usually falls between $-\pi$ and $+\pi$ radians, unless

219 crustal deformation exceeds a quarter of the wavelength which is $1.9 \mathrm{~m}$ in this case

220 study. However, crustal deformation in excess of $2 \mathrm{~m}$ was found during the 2016

221 Kumamoto Earthquake, and this is an extreme case that phase wraps of $\Delta \phi_{\text {diff appear. }}$

222 Even in such a case, unwrapping is not necessary by increasing number of divisions. 
223 We divided the chirp band into four bands (center frequency: $f_{1 \sim 4}$ ), and connected the

224 phase differences of interferometric phases for $\left(f_{4}-f_{3}\right),\left(f_{3}-f_{2}\right)$, and $\left(f_{2}-f_{1}\right)$. The phase

225 difference obtained for $f_{4}-f_{1}$ corresponded to $f_{\text {high }}-f_{\text {low }}$ in Eq. (3). Its frequency is 60

$226 \mathrm{MHz}$. The frequency of $f_{\mathrm{i}+1}-f_{\mathrm{i}}$ was $20 \mathrm{MHz}$ and corresponded to a wavelength of $15 \mathrm{~m}$,

227 and a phase wrap did not appear when the slant-range change was less than

228 approximately $3.75 \mathrm{~m}$; which was a quarter of the wavelength. Even if a larger

229 deformation occurred, it would have been possible to process the data without

230 unwrapping, by increasing the number of divisions further. The ionospheric delay

231 component was obtained by substituting the obtained phase difference ( $\left.\Delta \phi_{\text {diff }}\right)$ into Eq.

232 (3). As $\Delta \phi_{\text {disp }}$ is a wrapped phase, it was necessary to apply unwrapping. However, its

233 spatial distribution was smooth, except in extreme conditions, and it was easy to obtain

234 an appropriate unwrapped phase. From its unwrapped phase, the ionospheric delay

235 component was estimated by masking pixels with low coherence and applying a

236 Gaussian filter. Next, we simulated the atmospheric delay from the numerical weather

237 model (Ozawa and Shimizu, 2010, Ozawa et al., 2019) and removed it from the

238 interferograms of the four bands. Applying the same analysis used for the estimation of 
$239 \Delta \phi_{\text {diff }}$ to these interferograms, we obtained the unwrapped phase of the crustal

240 deformation component for $f_{4}-f_{1}$ from Eq. (2), without any unwrapping.

241 The slant-range change distributions obtained from the above analysis are shown in

242 Figs. 2(a) and 2(b), and the quasi-east-west (QEW) and quasi-up-down (QUD)

243 components converted using the method proposed by Fujiwara et al. (2000) are shown

244 in Fig. 3. Observation times for Path 28 and Path 126 differ by approximately half a

245 day, but it can be assumed that the crustal deformation that occurred during the time

246 difference in the observations is negligible because the maximum magnitude of the

247 earthquake that occurred during their time difference was MJMA4.2. QEW is a direction

248 rotating approximately $3^{\circ}$ clockwise from the east-west, and QUD is a direction

249 inclining approximately $8^{\circ}$ to the north from the vertical. In general, the results show an

250 eastward displacement in the northern area of the Futagawa Fault and a westward

251 displacement in the southern area, which is consistent with the focal mechanism of the

252 main shock. Its boundary clearly coincides with the Futagawa fault. The eastern edge of

253 the QEW boundary becomes obscure around the western rim of the Aso caldera, and in

254 the western area, the boundary extends to the Hinagu Fault from the Futagawa fault. On 
255 the other hand, the clear boundary of the QUD is observed in the western area of the

256 Futagawa Fault, while in the eastern area, the boundary is consistent with the

257 Idenokuchi Fault that is parallel to the Futagawa Fault (Figs. 3(f), (g)). Thus, the QEW

258 and QUD boundaries are different in this area, corresponding to the partitioning of a

259 strike-slip and a normal-slip, as described by Toda et al. (2016). Additionally, this study

260 clarified that subsidence gradually increased from the west, and that it exceeded $2 \mathrm{~m}$ in

261 the area between the Fugatawa and Idenokuchi faults. The maximum westward

262 displacement was also detected in this area, at $0.3-0.4 \mathrm{~m}$.

263 Crustal deformation west of the Aso caldera, where a complicated fringe pattern

264 appeared (Fig. 1(c)), was also clearly obtained (Figs. 3(d)-(g)). As pointed out by

265 Fujiwara et al. (2016), crustal deformation shows that a north-down dislocation

266 dominated in the southern area, and a south-down dislocation dominated in the north

267 area, similar to a graben. In addition, this result shows that the deformation gap was

268 large in the southern area. Clear deformation gaps can be observed at points where

269 surface ruptures have been found by field surveys (Awata et al., 2019; National Institute

270 of Advanced Industrial Science and Technology, 2019), and their extensions can also be 
271 identified in these results (thin lines in Fig. 3). Although the locations of gaps can be

272 also seen from the results of the conventional InSAR analysis, the DSI analysis is

273 advantageous because it can obtain also its amount.

274

\section{Discussions}

276 As described in the previous chapter, the case study on the application of DSI

277 analysis to the 2016 Kumamoto Earthquake yielded crustal deformation information

278 that was consistent with previous researches. Although new seismological knowledge

279 was not obtained in this case study, it was important to achieve reasonable results using

280 DSI analysis. Since seismological interpretations of crustal deformation have been

281 reported in many studies, we focus on the detection accuracy and limitations of the DSI

282 method in this study.

283 First, we compared the crustal deformation information obtained by the DSI analysis

284 with those from GEONET, which is the GNSS observation network operated by GSI

285 (Sagiya et al., 2000). Figure 4(a) shows the locations of the GNSS sites that were used

286 in this comparison. Crustal deformations from GNSS were derived from the averaged 
287 11-day difference coordinates of F3 solutions (Nakagawa et al., 2009) for 8 Apr. 2016

288 and 29 Apr. 2016. Because crustal deformation from GNSS included the effects of the

289 foreshock on April 14, crustal deformation calculated from the fault model (the two-

290 fault model with uniform slip), by Kobayashi (2017), were subtracted from it. Figure 4

291 shows the comparison between the crustal deformations obtained from the DSI analysis

292 and GEONET (these values are shown in Tables S1 and S2). The reference site for their

293 deformation is the "960699" GEONET site (Open diamond in Fig. 4(a)). With the

294 exception of the "960701" GEONET site, they agree within $10 \mathrm{~cm}$, and the root-mean-

295 squares of differences (rms) are $4.6 \mathrm{~cm}$ and $3.8 \mathrm{~cm}$ for Path 28 and Path 126,

296 respectively. Comparing the crustal deformations derived from conventional InSAR and

297 those from GEONET in the same way, the rms was $2.5 \mathrm{~cm}$ and $2.2 \mathrm{~cm}$ for Path 28 and

298 Path 126 (Tables S1 and S2), respectively, indicating that coincidence for the DSI

299 analysis is poorer than that for conventional InSAR. This is considered to arise from the

300 principal limitations of the DSI method. The SM1 mode of PALSAR-2 was observed

301 using a radar wave with a frequency of $1.2575 \mathrm{GHz}$, while the DSI analysis in this study

302 corresponds to an observation by a radar wave with a frequency of $60 \mathrm{MHz}$, indicating 
303 that the sensitivity of crustal deformation detection is much lower. Phase noise due to

304 decorrelation at almost the same level as conventional InSAR appears on each

305 interferogram for the divided band, but they are not compensated within the

306 interferograms of different frequencies. Decorrelation noise in the DSI result is

307 amplified $f_{0} /\left(f_{\text {high }}-f_{\text {low }}\right)$ times over that in conventional InSAR, corresponding to 21

308 times in this case study. Therefore, the accuracy of the DSI method is poorer than that

309 of conventional InSAR. In particular, for "960701" GEONET site, the slant-range

310 change results of the DSI analysis differ by more than $1 \mathrm{~m}$ from those from

311 conventional InSAR. This is much larger than the general accuracy of GNSS

312 observations, and therefore, a difference of $1 \mathrm{~m}$ is due to the error in the DSI analysis.

313 The coherence for this pixel was 0.04 , which is extremely low. In such pixels, the phase

314 difference between dividing bands is not appropriately estimated due to noise

315 amplification, and the errors may have arisen from the misestimation of the phase

316 difference $\Delta \phi_{\text {diff }}$ in Eq. (3).

317 To investigate the relationship between the coherence and accuracy of the DSI

318 method, we analyzed the post-seismic pair of Path 126 (April 29, 2016, and October 14, 
319 2016), where crustal deformation was much lower than that of the co-seismic pair. The

320 observation mode was the same as that for the co-seismic pair. Figures 5(a) and (b)

321 show slant-range change maps obtained using the DSI analysis and conventional

322 InSAR, and Fig. 5(c) shows the coherence map. In the slant range change map from the

323 DSI analysis, the area where high-frequency noise is dominant corresponds to the area

324 with low coherence. In the differences between the slant-range change maps obtained

325 from the DSI analysis and conventional InSAR, the deviations increased with

326 decreasing coherence (Fig. 5(d)). The standard deviation of the pixels with a coherence

327 of $0.7-0.8$ or larger was $2 \mathrm{~cm}$ or less. It increased with the decreases in coherence, and

328 for the pixels with a coherence of $0.5-0.6$, was estimated to be $3.5 \mathrm{~cm}$, corresponding to

329 the rms of the comparison with GEONET. An rms of approximately $10 \mathrm{~cm}$ was

330 obtained for a coherence of 0.2 , but the difference occasionally exceeded $1 \mathrm{~m}$ (Figs.

$3315(\mathrm{~d})$ and (e)). Noise increased significantly with decreases in coherence; thus, in DSI

332 analysis, SAR pairs with high coherence are necessary for the acquisition of highly

333 accurate of crustal deformation detections, and this method would be effectively

334 applicable to L-band SAR data that can obtain high coherence. Additionally, the noise 
due to the insufficient reduction of atmospheric delay was not amplified because it was

336 included as a nondispersive component. Noises due to insufficient reduction of

337 ionospheric delay are also not so different from that on the conventional InSAR.

338 Converting phase into the slant-range change, the ionospheric delay component is

$339 K / f_{\text {high }} / f_{\text {low }} \Delta$ TEC for the DSI method and $K / f_{0}{ }^{2} \Delta$ TEC for the conventional InSAR.

340 Next, the crustal deformation obtained from the DSI method was compared with that

341 from the offset-tracking method. We used the GAMMA SAR processor for the offset-

342 tracking analysis. In the co-registration of SAR images for the application of the offset-

343 tracking analysis, we corrected for the parallax topography effect due to the satellite

344 orbit difference and estimated the affine transformation coefficient by masking the areas

345 with large deformations. In the offset-tracking analysis, we set a correlation window of

$34664 \times 64$ pixels for every 24 pixels and estimated the offset with the maximum correlation.

347 Because crustal deformation can be estimated with higher spatial resolution by the use

348 of a smaller correlation window, we also attempted to perform an analysis using a

349 correlation window of $32 \times 32$ pixels. However, spike noise significantly affected these

350 results, and therefore, the results of the $64 \times 64$ correlation window was adopted for this 
comparison.

352 Figures 2(b) and (e) show the slant-range change maps obtained from the offset-

353 tracking analysis, and the differences with the results from the DSI analysis are shown

354 in Figs. 2(c) and (f). Generally, the slant-range change maps obtained by offset-tracking

355 analysis produced results very similar to those from the DSI analysis. Likewise, for the

356 comparison of the DSI and GEONET results, we compared slant-range changes from

357 the offset-tracking analysis with those from GEONET, and the rms of differences were

$3584.4 \mathrm{~cm}$ and $4.2 \mathrm{~cm}$ for Path 28 and Path 126 (Tables S1 and S2), respectively. These

359 values almost parallel the coincidence of the DSI analysis. However, granular patterns

360 with a wavelength of approximately several $100 \mathrm{~m}$ are superimposed over the entire

361 area on slant-range change maps from the offset-tracking analysis. Such crustal

362 deformation patterns are unnatural; and therefore, must be the result of estimation errors

363 in the offset-tracking analysis. Additionally, comparing the spatial distributions of

364 complicated deformations west of the Aso caldera, those from the DSI analysis are

365 sharper than that those from the offset-tracking analysis. This indicates that the DSI 
367 DSI analysis results, large amounts of random noise were obtained in the vicinity of the

368 fault. Additionally, a significantly negative difference from the offset tracking method

369 was also obtained near the fault. As coherence in this area was significantly low, it is

370 possible that crustal deformation was not correctly estimated in the DSI analysis. Thus,

371 it is possible that low coherence causes large misestimations in the slant-range changes

372 derived from DSI analysis.

373

374 Summary

375 In this study, we described the use of the DSI method for detecting huge crustal

376 deformations. In a case study, we applied the DSI method to PALSAR-2 data for the

3772016 Kumamoto Earthquake, and detected crustal deformation in the areas where the

378 unwrapped phase could not be obtained by conventional InSAR analysis, due to

379 complicated phase gaps. Compared with conventional InSAR, the detection accuracy of

380 the DSI method is poorer, but it can detect crustal deformation in areas where huge

381 crustal deformation has occurred. In addition, the DSI method can detect crustal

382 deformation with comparable accuracy and higher spatial resolution than the offset- 
383 tracking method. However, the detection accuracy of the DSI method depends on

384 coherence. An accuracy of approximately $2 \mathrm{~cm}$ was estimated for pixels with a

385 coherence of more than $0.7-0.8$, but an estimation error exceeding $1 \mathrm{~m}$ was occasionally

386 obtained for pixels with a coherence of less than 0.2 . The disadvantage of DSI analysis

387 is that applications with high accuracy are limited to cases that use high-coherence data

388 pairs. However, such a disadvantage will become insignificant in near future. New L-

389 band SAR missions, PALSAR-3/ALOS-4 and NISAR, will commence in the near

390 future, and observation frequency will be significantly improved from that of the current

391 PALSAR-2/ALOS-2. Since they will stably provide InSAR results with high coherence,

392 it is expected that the DSI method will become an effective tool for detection of huge

393 crustal deformations.

394

395 Declarations

396 Ethics approval and consent to participate

397 Not applicable.

398

399 Consent for publication 
$400 \quad$ Not applicable.

401

402 List of abbreviations

403 ALOS-2/4: Advanced Land Observing Satellite 2/4

404 DSI: Difference of Split-band Interferograms

405 EGM96: Earth Gravitational Model 1996

406 GEONET: GNSS Earth Observation Network System

407 GNSS: Global Navigation Satellite System

408 GSI: Geospatial Information Authority of Japan

409 InSAR: Interferometric SAR

410 JMA: Japan Meteorological Agency

411 JST: Japan standard time

412 NIED: National Research Institute for Earth Science and Disaster Resilience

413 NISAR: NASA-ISRO SAR

414 PALSAR-2: Phased Array type L-band SAR 2

415 QEW: Quasi-east-west

416 QUD: Quasi-vertical

417 SAR: Synthetic aperture radar

418 TEC: Total Electron Content 
421 ALOS-2/PALSAR-2 level 1.1 data can be purchased from RESTEC

422 (https://www.restec.or.jp/en/) or PASCO (http://en.alos-pasco.com).

423

424 Competing interests

425 The authors declare that they have no competing interests.

426

427 Funding

428 A part of this study was implemented in an ordinary research project of NIED. This

429 study partially supported by Specific Research Project (B) 2021-B-03 at Earthquake

430 Research Institute, the University of Tokyo.

431

432 Authors' contributions

433 TO developed the successful procedure of the DSI method and applied it to

434 PALSAR-2 data on the 2016 Kumamoto Earthquake. YH analyzed the same data set by

435 the offset-tracking method. All authors contributed to the preparation of the manuscript

436 and discussion of the results. 


\section{Acknowledgements}

439 We used ALOS-2/PALSAR-2 data that was shared within the PALSAR

440 Interferometry Consortium to Study our Evolving Land Surface (PIXEL) in this study.

441 The data were provided by the Japan Aerospace Exploration Agency (JAXA) under a

442 cooperative research contract with PIXEL. The ownership of original PALSAR-2 data

443 belongs to JAXA. GEONET data and the $10 \mathrm{~m}$-mesh digital elevation model published

444 by the GSI were used in this study. We used Generic Mapping Tools (Wessel and

445 Smith, 1998) to draw the figures.

446

447 Authors' information

448 National Research Institute for Earth Science and Disaster Resilience, 3-1 Tennodai

449 Tsukuba, Ibaraki, 305-0006, Japan

450 Taku Ozawa \& Yuji Himematsu

451

452 References

453 Awata, Y., Y. Shirahama, and Y. Kumahara (2019) (2) Integration of surface deformation

454 information associated with the 2016 Kumamoto earthquake in report of 

FY2016-2018”. Ministry of Education, Culture, Sports, Science and Technology

and

Kyushu

University https://www.jishin.go.jp/database/project_report/kumamoto_sogochousa/kumamoto _sogochousa-h28-h30/. Accessed 16 Sept 2021 (in Japanese, the title was translated to English by authors)

461

Brcic, R., A. Parizzi, M. Eineder, M., R. Bamler, and F. Meyer (2010) Estimation and compensation of ionospheric delay for SAR interferometry. in Proc. IEEE IGARSS $20102908-2911$.

465

Chen, C. W. and H. A. Zebker (2000) Network approaches to two-dimensional phase unwrapping: intractability and two new algorithms. J. Opt. Soc. Am. 17: 401-414.

468

Constantini, M. (1998) A novel phase unwrapping method based on network programming. IEEE Trans. Geosci. Remote Sensing 36(3): 813 - 821.

472 Fujiwara, S., T. Nishimura, M. Murakami,H. Nakagawa,M. Tobita, P. A. Rosen (2000) 2.5-D surface deformation of M6.1 earthquake near Mt Iwate detected by SAR interferometry. Geophys. Res. Lett. 27: 2049-2052. doi:10.1029/1999GL011291 

ruptures of the 2016 Kumamoto earthquake sequence detected by ALOS-2 SAR interferometry. Earth Planets Space 68:160. DOI:10.1186/s40623-016-0534-X

Gatelli, F., A. M. Guarnieri, F. Parizzi, P. Pasquali, C. Prati, and F. Rocca (1994) The wavenumber shift in SAR interferometry. IEEE Trasn. Geosci. Remote Sensing 32(4): 855-865. doi: $10.1109 / 36.298013$

Goldstein, R. M., H. Zebker, and C. L. Werner (1988) Satellite radar interferometry: Twodimensional phase unwrapping. Radio Sci. 23(4): 713-720.

Goldstein, R. M., and C. L. Werner (1998) Radar interferogram filtering for geophysical applications. Geophys. Res. Lett. 25: 4035-4038. doi: 10.1029/1998GL900033

Gomba, G., A. Parizzi, F. De Zan, M. Eineder, and R. Bamler (2016) Toward operational compensation of ionospheric effects in SAR interferograms: The split-spectrum method. IEEE Trans. Geosci. Remote Sens. 54(3) 1446-1461

Himematsu, Y., and M. Furuya (2016) Fault source model for the 2016 Kumamoto earthquake sequence based on ALOS-2/PALSAR-2 pixel-offset data: evidence for dynamic slip partitioning. Earth Planets Space 68:169. DOI:10.1186/s40623-016- 
500 Kobayashi, T. (2017) Earthquake rupture properties of the 2016 Kumamoto earthquake 501 foreshocks ( $\mathrm{M} \mathrm{j} 6.5$ and $\mathrm{M} \mathrm{j}$ 6.4) revealed by conventional and multiple-aperture 502 InSAR. Earth Planets Space 69:7. https://doi.org/10.1186/s40623-016-0594-y.

503

504 Lemoine, F. G., D. Smith, R. Smith, L. Kunz, E. Pavlis, N. Pavlis, S. Klosko, M. Chinn, 505 M. Torrence, R. Williamson, C. Cox, K. Rachlin, Y. Wang, S. Kenyon, R. Salman, R. 506 Trimmer, R. Rapp, and S. Nerem (1997) The development of the NASA GSFC and $507 \quad$ NIMA Joint Geopotential Model. in Gravity, Geoid, and Marine Geodesy. Segawa J 508 et al. (eds) IAG Symp.: vol 117, Springer, New York, 461-469

509

510 Madsen, S. N. and H. A. Zebker (1992) Automated Absolute Phase Retrieval in AcrossTrack Interferometry. in proc. IGARSS'92 2:1582-1584. 10.1109/IGARSS.1992.578639

514 Nakagawa, H, T. Toyofuku K. Kotani, B. Miyahara, C. Iwashita, S. Kawamoto, Y. 515 Hatanaka, H. Munekane, M. Ishimoto, T. Yutsudo, N. Ishikura, Y. Sugawara (2009) 516 Development and validation of GEONET New Analysis Strategy (version 4). J Geogr 517 Surv Inst 118:1-8. (in Japanese)

519 National Institute of Advanced Industrial Science and Technology (2019) Active Fault Database of Japan, February 28, 2012 version, Research Information Database DB095, 
521 National Institute of Advanced Industrial Science and Technology. 522 https://gbank.gsj.jp/activefault/. Accessed 16 Sept 2021

523

524 Okada Y., K. Kasahara, S. Hori, K. Obara, S. Sekiguchi, H. Fujiwara, A. Yamamoto 525 (2004) Recent progress of seismic observation networks in Japan Hi-net, F-net, K526 NET and KiK-net. Earth Planets Space 56 XV-XXViii. doi:10.1186/BF03353076

528 Ozawa, T. and S. Shimizu (2010) Atmospheric noise reduction in InSAR analysis using 529 numerical weather model. J. Geod. Soc. Jpn 56:137-147. 530 doi:10.11366/sokuchi.56.137 (in Japanese with English abstract)

532 Ozawa, T., E. Fujita, and H. Ueda (2016) Crustal deformation associated with the 2016 533 Kumamoto Earthquake and its effect on the magma system of Aso volcano. Earth $534 \quad$ Planets Space 68:186. DOI:10.1186/s40623-016-0563-5

536 Ozawa, T., Y. Aoki, S. Okuyama, X. Wang, Y. Miyagi, and A. Nohmi (2019) Database of 537 crustal deformation observed by SAR: Improving atmospheric delay mitigation for 538 satellite SAR interferometry and developing L-Band multi-type portable SAR. J. 539 Disaster Res. 14(5):713-727

541 Rosen, P., S. Hensley, and C. Chen (2010) Measurement and mitigation of the ionosphere in L-band interferometric SAR data. in Proc. IEEE Radar Conf. May 2010. 1459- 
545 Sagiya, T., S. Miyazaki, and T. Tada (2000) Continuous GPS array and present-day crustal 546 deformation of Japan. Pure Appl. Geophys. 157: 2303-2322

548 Shirahama, Y., M. Yoshimi, Y. Awata, T. Maruyama, T. Azuma, Y. Miyashita, H. Mori, K.

549 Imanishi, N. Takeda, T. Ochi, M. Otsubo, D. Asahina, and A. Miyakawa (2016) 550 Characteristics of the surface ruptures associated with the 2016 Kumamoto earthquake 551 sequence, central Kyushu, Japan. Earth Planets Space 68:191. DOI:10.1186/s40623552 016-0559-1

553

554 Toda, S., H. Kaneda, S. Okada, D. Ishimura, and Z. K. Mildon (2016) Slip-partitioned 555 surface ruptures for the Mw 7.0 16 April 2016 Kumamoto, Japan, earthquake. Earth 556 Planets Space 68:188. DOI:10.1186/s40623-016-0560-8

557

558 Wegmüller, U., C. Werner, O. Frey, C. Magnard, and T. Strozzi (2018) Estimation and 559 compensation of the ionospheric phase in SAR interferograms. Procedia Computer $560 \quad$ Sci. $138: 318-325$

561

562 Wessel P., and W. H. F. Smith (1998) New improved version of generic mapping tools

563 released. EOS Trans AGU 79(47):579. doi:10.1029/98EO00426. 
(a)
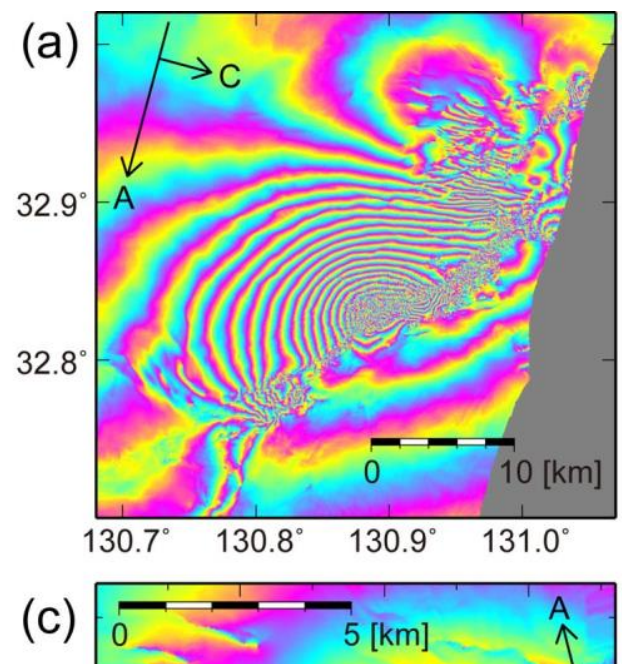

$32^{\circ} 58^{\prime}$
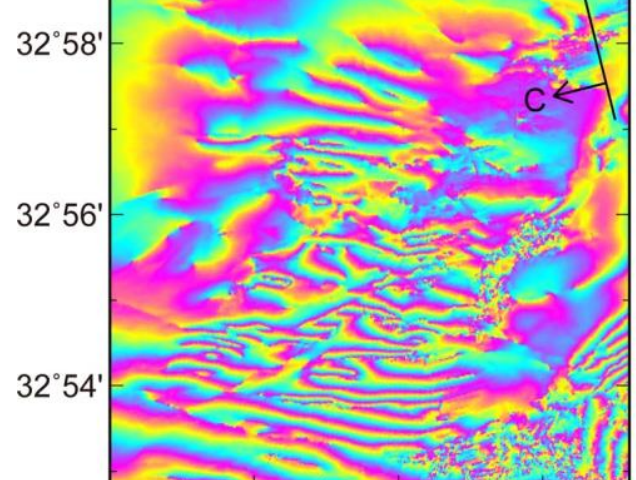

$130^{\circ} 56^{\prime} \quad 130^{\circ} 58^{\prime} \quad 131^{\circ} 00^{\prime}$

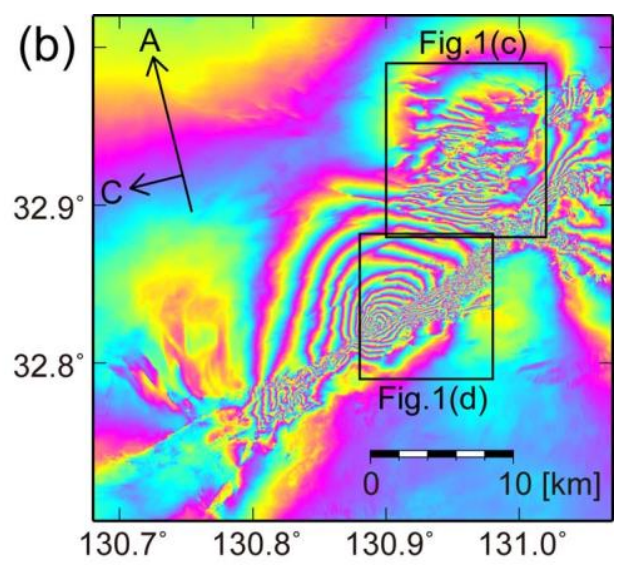

(d)
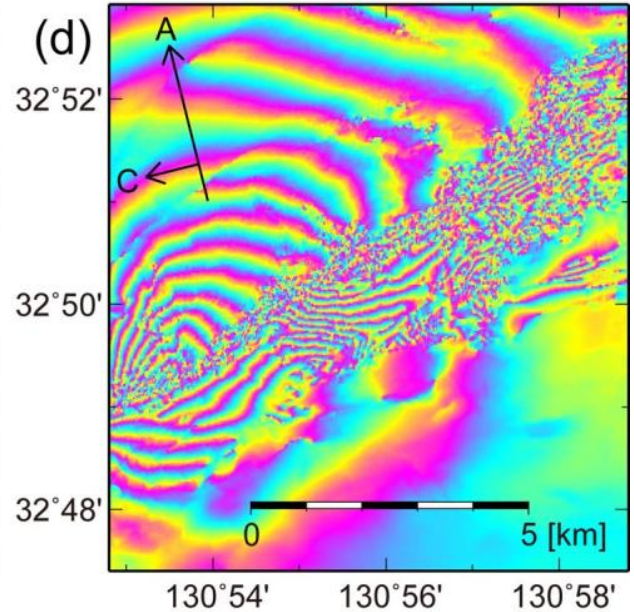

$+2 \pi$

$(+12 \mathrm{~cm})$

567 Figure 1. PALSAR-2 interferograms depicting the crustal deformation associated with the 5682016 Kumamoto Earthquake. Arrows in the figures indicate the along-track (A) and 569 cross track (C) directions. (a) Interferogram for Path 28 (Descending, Left looking). 570 (b) Interferogram for Path 126 (Ascending, Left looking). The two boxes correspond 571 to the areas of (c) and (d). (c) Interferogram for Path 28, enlarged around the northeast 572 of the Aso caldera. (d) Interferogram for Path 28, enlarged around the Futagawa Fault. 

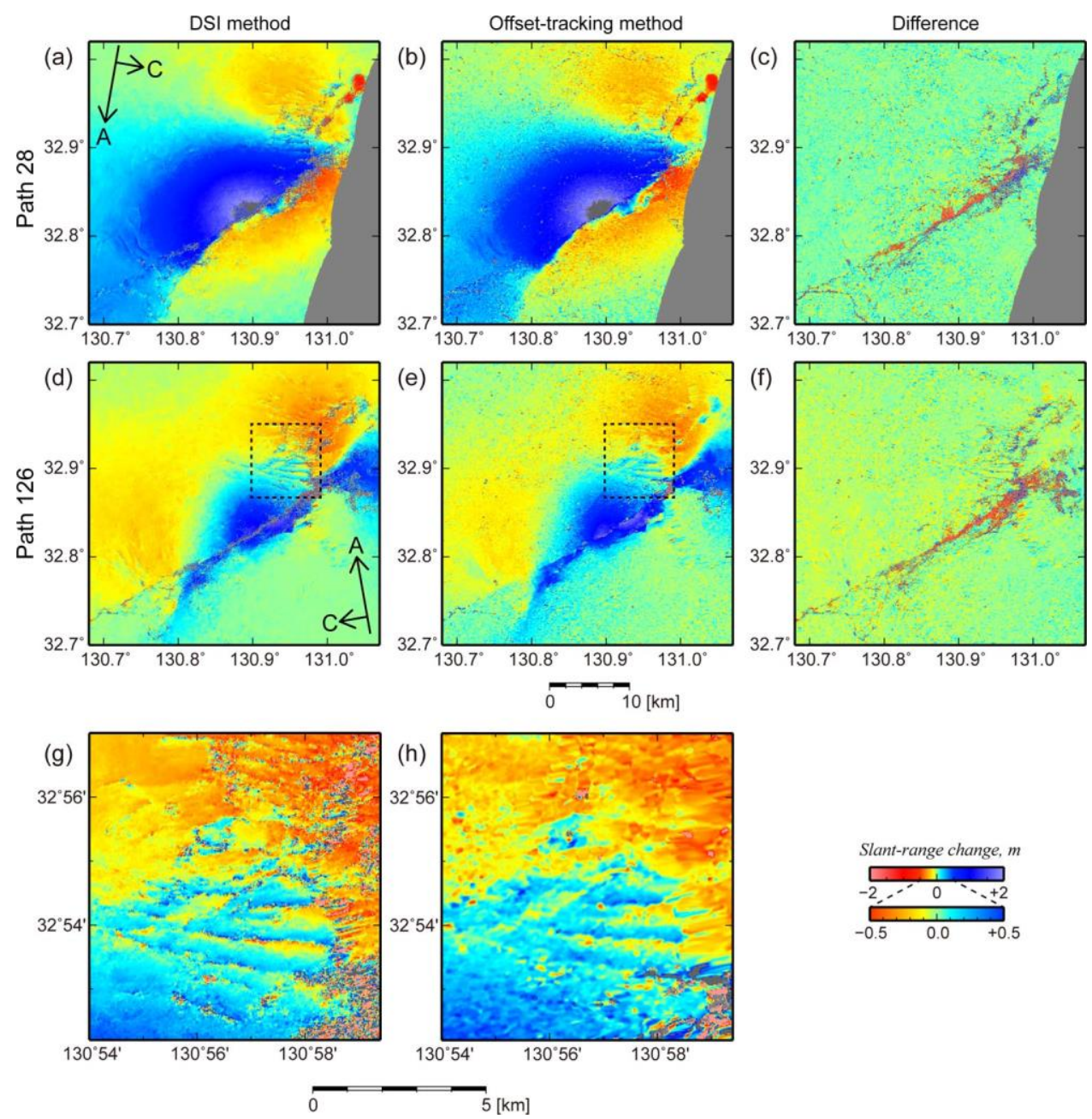

575 Figure 2. Slant-range change maps obtained from the DSI and the offset-tracking methods 576 and the differences between them. Arrows in the figures indicate the along-track (A) and cross track (C) directions. (a) Slant-range change map for Path 28, using the DSI method. (b) Slant-range change map for Path 28, using the offset-tracking method. (c) Differences between the slant-range change maps, using the DSI and offset-tracking methods for Path 28. (d) Slant-range change map for Path 126, using the DSI method. Dashed box corresponds to the area shown in (g) and (h). (e) Slant-range change map for Path 126, using the offset-tracking method. (f) Difference of slant-range change maps, using the DSI and offset-tracking methods for Path 126. (g) Enlarged slantrange change map, using the DSI method for Path 126. The area corresponds to dashed area in (d). (h) Enlarged slant-range change map by the offset-tracking method for Path 126. The area depicted corresponds to the dashed area in (e). 

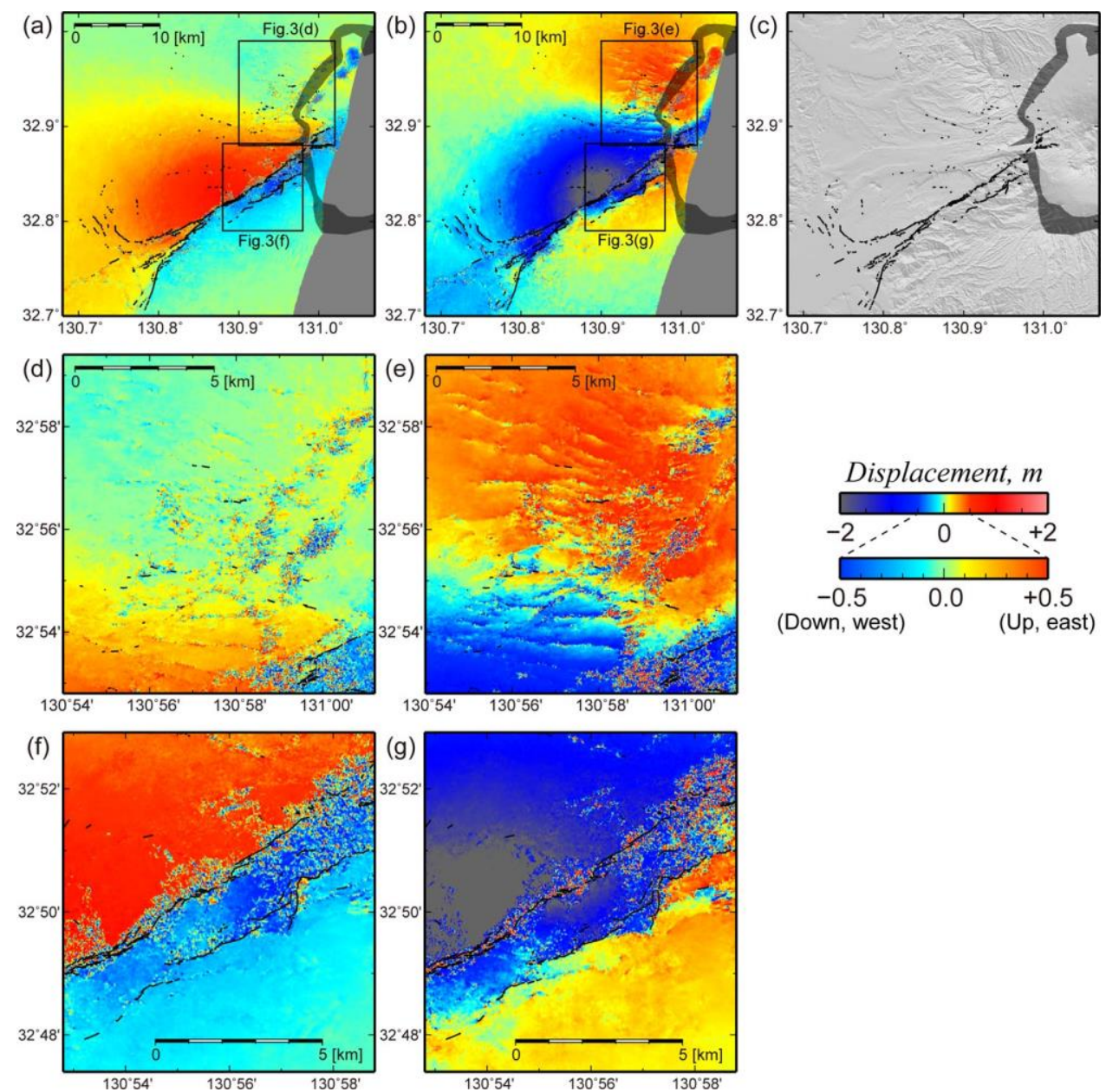

Figure 3. Two-dimensional crustal deformation calculated from the slant-range changes for Paths 28 and 126. Thin lines depict the surface ruptures associated with the 2016 Kumamoto Earthquake (Awata et al., 2019; National Institute of Advanced Industrial Science and Technology, 2019). (a) Quasi-east-west (QEW) component of crustal deformation. It is a component rotated 3 degrees clockwise from East. The two boxes correspond to the areas of (d) and (f). Shaded areas show the caldera wall. (b) Quasiup-down (QUD) component of crustal deformation. It is a component that inclines 8 degrees to the north direction from the vertical. The two boxes correspond to the areas in (e) and (g). (c) Topographic relief map. (d) Distribution of the QEW component enlarged about the northeast of the Aso caldera, corresponding to the Fig. 1(c). (e) Distribution of the QUD component, enlarged in the northeast of the Aso caldera, corresponding to Fig. 1(c). (e) Distribution of the QEW component, enlarged around the Futagawa Fault, corresponding to Fig. 1(d). (e) Distribution of the QUD component, enlarged around the Futagawa Fault, corresponding to Fig. 1(d). 

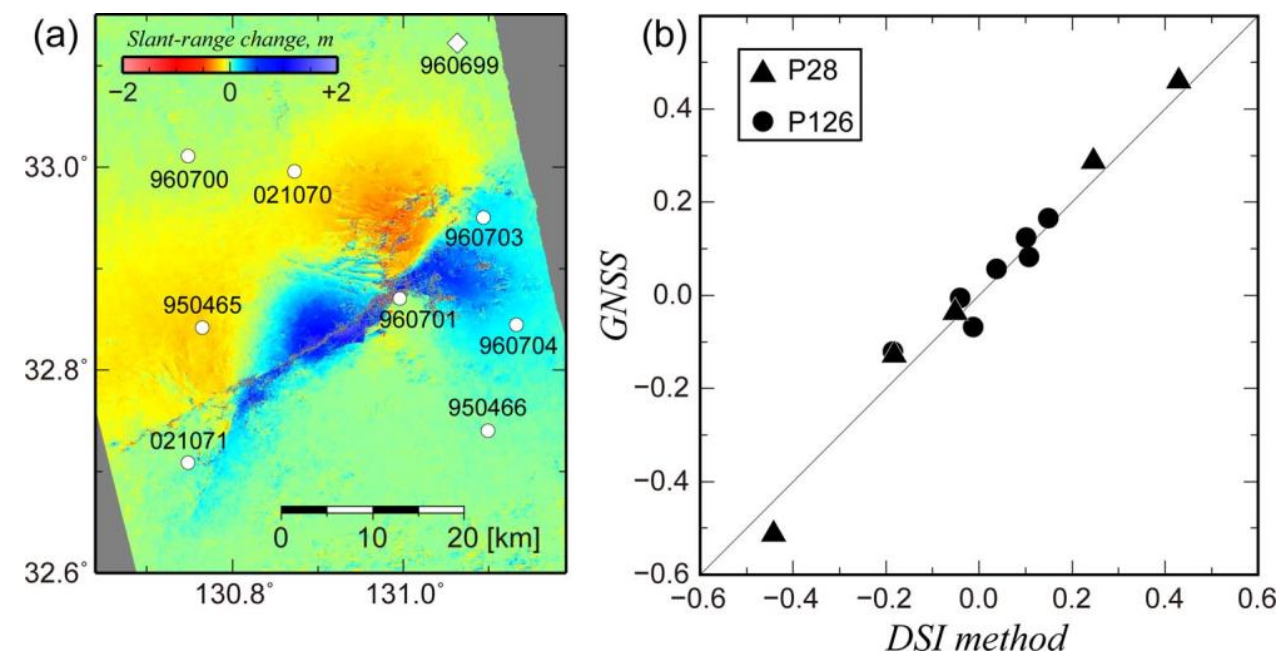

603

604 Figure 4. (a) Distribution of the GEONET stations used in the comparison of slant-range 605 changes. The background depicts the slant-range change maps for Path 126, using the 606 DSI method. Open diamond indicates GEONET station "960699", the reference point 607 of crustal deformation. (b) Comparison of slant-range changes obtained from the DSI 608 method and GEONET. Triangles and circles indicate the slant-range changes for Path 60928 and Path 126, respectively. 

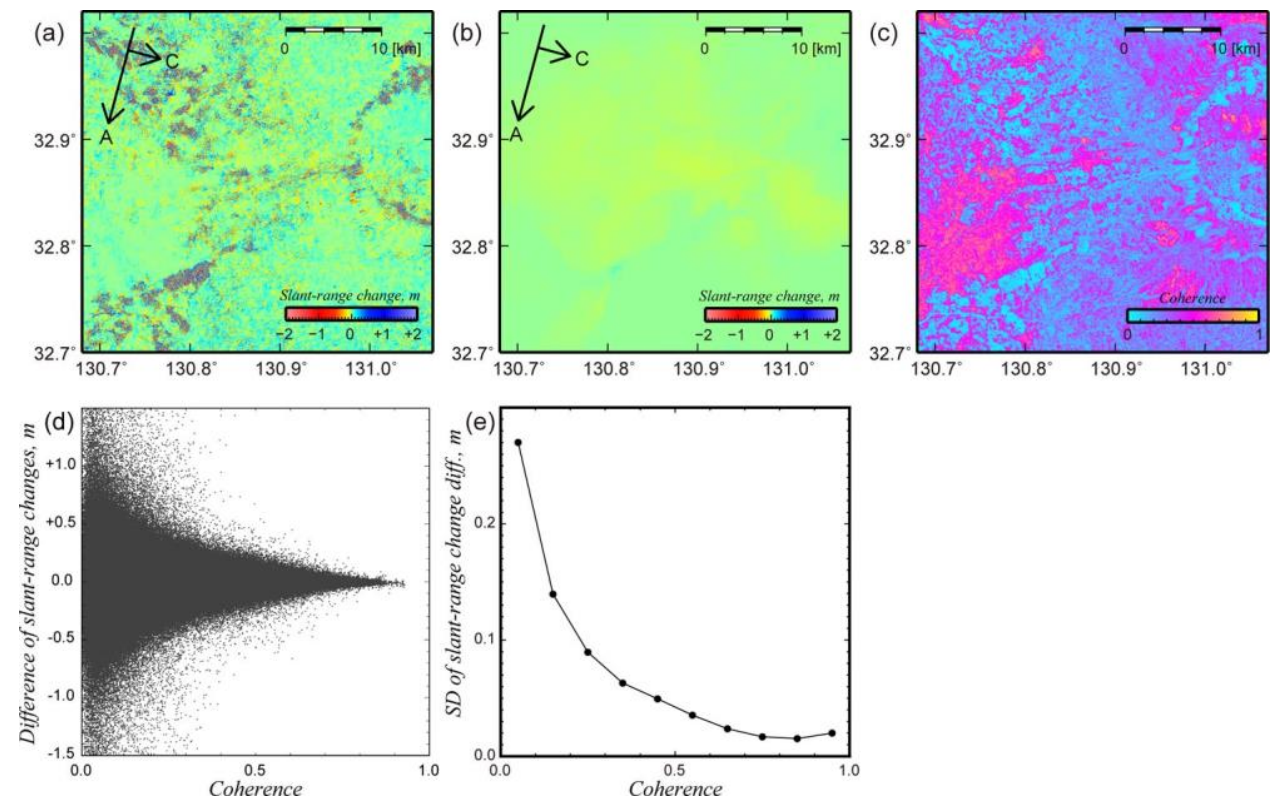

612 Figure 5. (a) The slant-range change map about the post-seismic pair of Path 126,

613 obtained using the DSI method. (b) The slant-range change map from conventional 614 InSAR. (c) Coherence map. (d) Scatter plot of difference of slant-range changes 615 obtained using the DSI (y-axis) versus coherences (x-axis). (e) The diagram depicts 616 the relationship between the coherence and standard deviation of the differences in 617 the slant-range changes. 
619 Table S1. Comparison of the slant-range changes from GNSS, DSI, InSAR, and offset-

620 tracking for Path 28.

\begin{tabular}{|c|c|c|c|c|c|}
\hline Site & GNSS $[\mathrm{m}]$ & DSI $[\mathrm{m}]$ & InSAR $[\mathrm{m}]$ & Off-track [m] & Coherence \\
\hline 021070 & -0.129 & -0.182 & -0.144 & -0.224 & 0.38 \\
\hline 021071 & 0.288 & 0.246 & 0.254 & 0.307 & 0.40 \\
\hline 950465 & 0.460 & 0.430 & 0.434 & 0.488 & 0.50 \\
\hline 950466 & - & - & - & - & - \\
\hline 960700 & -0.037 & -0.052 & -0.056 & -0.016 & 0.41 \\
\hline 960701 & -0.513 & -0.442 & -0.488 & -0.555 & 0.11 \\
\hline 960703 & - & - & - & - & - \\
\hline 960704 & - & - & - & - & - \\
\hline 960699 & & \multicolumn{2}{|c|}{ Reference site } & & 0.45 \\
\hline rms* & & 0.046 & 0.025 & 0.044 & \\
\hline
\end{tabular}

$622 *$ The root-mean-square of differences in slant-range changes from GNSS.

623 
624 Table S2. Comparison of the slant-range changes from GNSS, DSI, InSAR, and offset-

625 tracking for Path 126.

\begin{tabular}{|c|c|c|c|c|c|}
\hline Site & GNSS [m] & DSI $[\mathrm{m}]$ & InSAR $[\mathrm{m}]$ & Off-track [m] & Coherence \\
\hline 021070 & -0.067 & -0.013 & -0.071 & -0.029 & 0.31 \\
\hline 021071 & 0.083 & 0.107 & 0.041 & 0.073 & 0.36 \\
\hline 950465 & -0.120 & -0.185 & -0.154 & -0.133 & 0.56 \\
\hline 950466 & 0.057 & 0.038 & 0.044 & 0.003 & 0.44 \\
\hline 960700 & -0.005 & -0.040 & -0.017 & -0.038 & 0.47 \\
\hline 960701 & 0.137 & -1.294 & 0.058 & -0.019 & 0.04 \\
\hline 960703 & 0.166 & 0.149 & 0.151 & 0.105 & 0.53 \\
\hline 960704 & 0.125 & 0.102 & 0.121 & 0.062 & 0.63 \\
\hline 960699 & & \multicolumn{2}{|c|}{ Reference site } & & 0.55 \\
\hline rms* & & 0.038 & 0.022 & 0.042 & \\
\hline
\end{tabular}

$627 *$ The root-mean-square of the differences in slant-range changes from GNSS without 628 “960701”. 


\section{Supplementary Files}

This is a list of supplementary files associated with this preprint. Click to download.

- TableS1.xlsx

- TableS2.xIsx

- graphicalabstract.jpg 